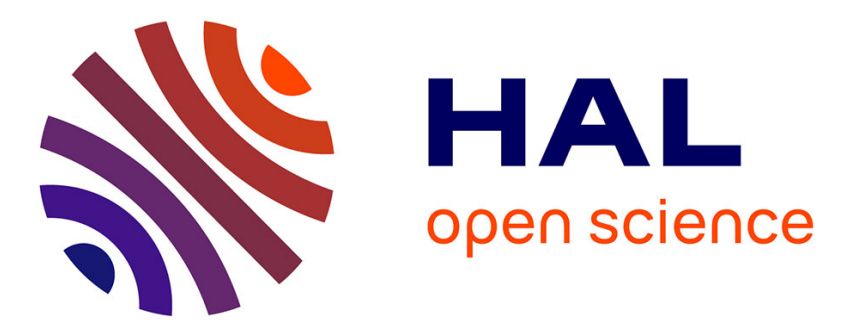

\title{
Seismic Wave Propagation Simulations on Low-power and Performance-centric Manycores
}

Márcio Castro, Emilio Francesquini, Fabrice Dupros, Hideo Aochi, Philippe

Navaux, Jean-François Mehaut

\section{To cite this version:}

Márcio Castro, Emilio Francesquini, Fabrice Dupros, Hideo Aochi, Philippe Navaux, et al.. Seismic Wave Propagation Simulations on Low-power and Performance-centric Manycores. Parallel Computing, 2016, 10.1016/j.parco.2016.01.011 . hal-01273153

\section{HAL Id: hal-01273153 \\ https://hal.science/hal-01273153}

Submitted on 12 Feb 2016

HAL is a multi-disciplinary open access archive for the deposit and dissemination of scientific research documents, whether they are published or not. The documents may come from teaching and research institutions in France or abroad, or from public or private research centers.
L'archive ouverte pluridisciplinaire $\mathbf{H A L}$, est destinée au dépôt et à la diffusion de documents scientifiques de niveau recherche, publiés ou non, émanant des établissements d'enseignement et de recherche français ou étrangers, des laboratoires publics ou privés. 


\title{
Seismic Wave Propagation Simulations on Low-power and Performance-centric Manycores
}

\author{
Márcio Castro ${ }^{\mathrm{a}}$, Emilio Francesquini $^{\mathrm{b}}$, Fabrice Dupros $^{\mathrm{c}}$, Hideo Aochi $^{\mathrm{c}}$, \\ Philippe O. A. Navaux ${ }^{\mathrm{d}}$, Jean-François Méhaut ${ }^{\mathrm{e}}$ \\ ${ }^{a}$ Department of Informatics and Statistics, Federal University of Santa Catarina (UFSC) \\ Campus Reitor João David Ferreira Lima, Trindade, 88040-970, Florianópolis, Brazil \\ ${ }^{b}$ Institute of Computing, University of Campinas (UNICAMP) \\ Av. Albert Einstein, 1251, Cidade Universitária, 13083-852, Campinas, Brazil \\ ${ }^{c} B R G M$ \\ BP 6009, 45060 Orléans Cedex 2, France \\ ${ }^{d}$ Informatics Institute, Federal University of Rio Grande do Sul (UFRGS) \\ Av. Bento Gonçalves, 9500, Campus do Vale, 91501-970, Porto Alegre, Brazil \\ ${ }^{e}$ CEA-DRT - LIG Laboratory, University of Grenoble Alpes \\ 110 Avenue de la Chimie, 38400 Saint-Martin d'Hères, France
}

\begin{abstract}
The large processing requirements of seismic wave propagation simulations make High Performance Computing (HPC) architectures a natural choice for their execution. However, to keep both the current pace of performance improvements and the power consumption under a strict power budget, HPC systems must be more energy efficient than ever. As a response to this need, energyefficient and low-power processors began to make their way into the market. In this paper we employ a novel low-power processor, the MPPA-256 manycore, to perform seismic wave propagation simulations. It has 256 cores connected by a NoC, no cache-coherence and only a limited amount of on-chip memory. We describe how its particular architectural characteristics influenced our solution for an energy-efficient implementation. As a counterpoint to the low-power MPPA-256 architecture, we employ Xeon Phi, a performance-centric manycore. Although both processors share some architectural similarities, the challenges to implement an efficient seismic wave propagation kernel on these platforms are very different. In this work we compare the performance and energy efficiency of our implementations for these processors to proven and optimized solutions for other hardware platforms such as general-purpose processors and a GPU. Our experimental results show that MPPA-256 has the best energy efficiency, consuming at least $77 \%$ less energy than the other evaluated platforms, whereas the performance of our solution for the Xeon Phi is on par with a state-of-the-art solution for GPUs.
\end{abstract}

Keywords: Seismic wave propagation; MPPA-256; Xeon Phi; HPC; performance; energy efficiency

Email addresses: marcio.castro@ufsc.br (Márcio Castro) francesquini@ic.unicamp.br (Emilio Francesquini), f.dupros@brgm.fr (Fabrice Dupros), 


\section{Introduction}

Public policies for risk mitigation and damage assessment in hypothetical earthquakes scenarios as well as oil and gas exploration make an intensive use of simulations of large-scale seismic wave propagation. In order to provide realistic results, these simulations rely on complex models, which often demand considerable computational resources to reliably process vast amounts of data in a reasonable amount of time.

Such processing capabilities are often provided by High Performance Computing (HPC) platforms. Up until recently, HPC platform capabilities were evaluated almost exclusively based on their raw processing speed. However, one of the aspects that hinder the quest for ever growing performance is the excessive use of energy. For that reason, the energy efficiency of HPC platforms has become, in some contexts, as important as their raw performance.

Indeed, the seek for alternatives to lower current energy consumption arose first in the embedded systems community but lately became one of the major concerns of the HPC scientific community $[1,2]$. Recently, manycore processors, a new class of highly-parallel chips, was unveiled. Tilera Tile-Gx [3], Kalray MPPA-256 [4], Adapteva Epiphany-IV [5], Intel Single-Chip Cloud Computer (SCC) [6] and Xeon Phi are examples of such processors, providing up to hundreds of autonomous cores that can be used to accomplish both data and task parallelism. This distinctive characteristic sets them apart from SIMD-like highly-parallel architectures such as Graphics Processing Units (GPUs). For this reason, in this paper, we classify GPUs separately, in their own category.

While some manycore processors may present better energy efficiency than state-of-the-art general-purpose multicore processors [7], their particular architectural characteristics make the development of efficient scientific parallel applications a challenging task $[5,8]$. Some of these processors are built and optimized for certain classes of embedded applications like signal processing, video decoding and routing. Additionally, processors such as MPPA-256 have important memory constraints, e.g., limited amount of directly addressable memory and absence of cache coherence protocols. Furthermore, communication costs between cores are not uniform, they depend on the location of the communicating cores. One of the main reasons for this difference in communication cost is the Network-on-Chip (NoC). When applications distribute work among the cores of the processor, they should take into consideration the placement of these tasks and their communication patterns to minimize communication costs. This problem is similar to that faced by applications running on NUMA platforms [9, 10], or MPI applications that try to take into account both the network topology and the memory hierarchy to improve performance [11, 12].

In this paper we outline the architectural distinctiveness of the low-power MPPA-256 manycore processor. Considering these characteristics, we describe how the main kernel of a seismic wave propagation simulator was adapted to

h. aochi@brgm.fr (Hideo Aochi), navaux@inf .ufrgs.br (Philippe O. A. Navaux), jean-francois.mehaut@imag.fr (Jean-François Méhaut) 
this platform. Due to the limited size of the local memories in MPPA-256, we developed a new multi-level tiling strategy and a prefetching mechanism to allow us to deal with real simulation scenarios and to alleviate communication overheads. We also describe the difficulties and solutions (some of them generic enough to be used in different contexts) we employed during this adaptation.

As a counterpoint to the low-power MPPA-256, we also adapted the same kernel to a performance-centric manycore, the Xeon Phi. Although both processors share some architectural characteristics such as the presence of a NoC, non-uniform costs of communication between cores and limited fast local memories, they have their own peculiarities which directed us to employ different approaches in each case. For instance, in both architectures, each thread has access to approximately $128 \mathrm{kB}$ of fast local memory. However, their memory organization is very different. On Xeon Phi each core has $512 \mathrm{kB}$ of L2 shared by up to 4 threads. On the other hand, on MPPA-256 the processing cores have $8 \mathrm{kB}$ of L1 cache each and cores are grouped into clusters of 16. Each one of these clusters has $2 \mathrm{MB}$, of work memory shared among the cores. Contrary to Xeon Phi, MPPA-256 does not have cache-coherence or automatic load/store of values from/to main memory, and all memory transferences must be handled by the programmer explicitly. Ultimately, this means that in order to be able to achieve good performance on MPPA-256, the application is obliged to tightly control communications between the cores in the same cluster, between clusters, and between each cluster and the main memory. This paper explains the details that made us decide for a multi-level tiling strategy proposed for MPPA-256 instead of a more traditional approach of cache blocking that was used for Xeon Phi.

Taking as a basis optimized GPU and general-purpose processor implementations for this kernel, we show that even if MPPA-256 presents an increased software development complexity, it can indeed be used as an energy-efficient alternative to perform seismic wave propagation simulations. Our results show that the solution we propose for the MPPA-256 processor achieves the best energy efficiency, consuming $77 \%, 86 \%$ and $88 \%$ less energy than optimized solutions for GPU, general-purpose and Xeon Phi architectures, respectively. The performance achieved on Xeon Phi, on the other hand, is comparable to a stateof-the-art GPU. Moreover, the execution was $58 \%$ and $73 \%$ faster than that of multicores and MPPA, respectively.

The remainder of this paper is organized as follows. Section 2 discusses the fundamentals of seismic wave propagation. Then, Section 3 discusses the main challenges we must overcome when dealing with parallel seismic wave propagations on MPPA-256 and Xeon Phi. Moreover, it presents our approaches to perform efficient seismic wave propagation simulations on both processors. Section 4 discusses performance and energy efficiency experimental results. Section 5 describes related work and Section 6 concludes this paper.

\section{Seismic Wave Propagation}

Quantitative earthquake hazard assessment is crucial for public policy, reduction of future damages and urban planning. Recent important geological 
events, such as the $\mathrm{M}_{\mathrm{W}} 7.9$ (moment magnitude) earthquake in China (2008) or the $\mathrm{M}_{\mathrm{W}} 8.8$ earthquake in Chile (2010), have been studied using numerical tools with good agreement with observation. From a physical point a view, the mechanisms used to describe earthquake events are complex and highly nonlinear. For the sake of simplicity, wave propagation simulations usually consider elastic domains and include some anelasticity to account for attenuations. However, in the case of strong motion, these simplifying assumptions are not capable of correctly describing the phenomenon. A fundamental challenge for earthquake engineering is predicting the level and variability of strong ground motion from future earthquakes. Enhancing this predictive ability requires understanding of the earthquake source, the effects of the propagation path on the seismic waves, and basin and near-surface site effects.

In this paper, we restrict our discussion to the elastodynamics equations corresponding to the elastic situation using a linear model. However, our approach could also be used in nonlinear models that rely on a linear solution inside the iterative procedure such as Newton-like methods. Among numerical methods, variational methods such as finite element method [14] or spectral element method [15] can be employed for both regional and global scale seismology whereas the Discontinous Galerkin method is very efficient for earthquake source modeling [16]. In terms of popularity, the Finite Difference Method (FDM) remains one of the top contenders due to its implementation simplicity and efficiency for a wide range of applications. Although this method was initially proposed almost thirty years ago [17], this classic discretization technique has been the focus of continuous refinements since its proposal [18, 19].

Simulations of seismic wave propagations are often constrained by the computational and storage capacity of the hardware platform. Thus seismologists often reduce the scope of the simulation to a small volume instead of considering the whole Earth. The memory footprint of each simulation depends on both the domain of interest and the number of grid points per wavelength. This last condition guarantees numerical stability and therefore the accuracy of the simulation. In our case, for practical reasons, we limit our simulations to problem domains that fit into the $2 \mathrm{~GB}$ of memory available on the MPPA-256 platform. For that, we perform regional scale modeling spanning a few hundred kilometers in each spatial direction.

In the next section we present the standard sequential algorithm for seismic wave propagation simulations. Aochi et al. [20] provide a more detailed description of the equations governing these simulations in the case of an elastic material.

\subsection{Discretization and Standard Sequential Algorithm}

As mentioned before, the FDM is one of the most popular techniques to solve the elastodynamics equations and to simulate the propagation of seismic waves. One of the key features of this scheme is the introduction of a staggeredgrid [17] for the discretization of the seismic wave equation, which in the case of an elastic material is: 


$$
\rho \frac{\partial v_{i}}{\partial t}=\frac{\partial \sigma_{i j}}{\partial j}+F_{i}
$$

$$
\frac{\partial \sigma_{i j}}{\partial t}=\lambda \delta_{i j}\left(\frac{\partial v_{x}}{\partial x}+\frac{\partial v_{y}}{\partial y}+\frac{\partial v_{z}}{\partial z}\right)+\mu\left(\frac{\partial v_{i}}{\partial j}+\frac{\partial v_{j}}{\partial i}\right)
$$

Where indices $i, j, k$ represent a component of a vector or tensor field in cartesian coordinates $(x, y, z), v_{i}$ and $\sigma_{i j}$ represent the velocity and stress field respectively, and $F_{i}$ denotes an external source force. $\rho$ is the material density and $\lambda$ and $\mu$ are the elastic coefficients known as Lamé parameters. A time derivative is denoted by $\frac{\partial}{\partial t}$ and a spatial derivative with respect to the $i$-th direction is represented by $\frac{\partial}{\partial i}$. The Kronecker symbol $\delta_{i j}$ is equal to 1 if $i=j$ and zero otherwise.

Indeed, all the unknowns are evaluated at the same location for classical collocated methods over a regular Cartesian grid whereas the staggered grid leads to a shift of the derivatives by half a grid cell. The equations are rewritten as a first-order system in time and therefore the velocity and the stress fields can be simultaneously evaluated at a given time step.

Exponents $i, j, k$ indicate the spatial direction with $\sigma^{i j k}=\sigma(i \Delta s, j \Delta s, k \Delta s)$, $\Delta s$ corresponds to the space step and $\Delta t$ to the time step. The elastic coefficients $\rho, \mu$ et $\lambda$ are defined at location $(i, j, k)$. Starting at time step $t=\ell \Delta t$, the following unknowns corresponding to the next time step $t=\left(\ell+\frac{1}{2}\right) \Delta t$ are computed:

$$
v_{x}^{\left(i+\frac{1}{2}\right) j k}\left(l+\frac{1}{2}\right), \quad v_{y}^{i\left(j+\frac{1}{2}\right) k}\left(l+\frac{1}{2}\right), \quad v_{z}^{i j\left(k+\frac{1}{2}\right)}\left(l+\frac{1}{2}\right)
$$

Then, at time $t=(\ell+1) \Delta t$, the following unknowns are computed:

$$
\begin{aligned}
& \sigma_{x x}^{i j k}(l+1), \quad \sigma_{y y}^{i j k}(l+1), \quad \sigma_{z z}^{i j k}(l+1), \quad \sigma_{x y}^{\left(i+\frac{1}{2}\right)\left(j+\frac{1}{2}\right) k}(l+1), \\
& \sigma_{z y}^{i\left(j+\frac{1}{2}\right)\left(k+\frac{1}{2}\right)}(l+1), \quad \sigma_{z x}^{\left(i+\frac{1}{2}\right) j\left(k+\frac{1}{2}\right)}(l+1)
\end{aligned}
$$

For instance, the stencil applied for the computation of the velocity component in the $x$-direction is given by:

$$
\begin{aligned}
& v_{x}^{\left(i+\frac{1}{2}\right) j k}\left(l+\frac{1}{2}\right)=v_{x}^{\left(i+\frac{1}{2}\right) j k}\left(l-\frac{1}{2}\right)+a_{1} F_{x}^{\left(i+\frac{1}{2}\right) j k} \\
& \quad+a_{2}\left[\frac{\sigma_{x x}^{(i+1) j k}-\sigma_{x x}^{i j k}}{\Delta x}+\frac{\sigma_{x y}^{\left(i+\frac{1}{2}\right)\left(j+\frac{1}{2}\right) k}-\sigma_{x y}^{\left(i+\frac{1}{2}\right)\left(j-\frac{1}{2}\right) k}}{\Delta y}+\frac{\sigma_{x z}^{\left(i+\frac{1}{2}\right) j\left(k+\frac{1}{2}\right)}-\sigma_{x z}^{\left(i+\frac{1}{2}\right) j\left(k-\frac{1}{2}\right)}}{\Delta z}\right] \\
& \quad-a_{3}\left[\frac{\sigma_{x x}^{(i+2) j k}-\sigma_{x x}^{(i-1) j k}}{\Delta x}+\frac{\sigma_{x y}^{\left(i+\frac{1}{2}\right)\left(j+\frac{3}{2}\right) k}-\sigma_{x y}^{\left(i+\frac{1}{2}\right)\left(j-\frac{3}{2}\right) k}}{\Delta y}+\frac{\sigma_{x z}^{\left(i+\frac{1}{2}\right) j\left(k+\frac{3}{2}\right)}-\sigma_{x z}^{\left(i+\frac{1}{2}\right) j\left(k-\frac{3}{2}\right)}}{\Delta z}\right]
\end{aligned}
$$


The computational procedure representing this evaluation is described in Algorithm 2.1. Inside the time step loop, the first triple nested loop is devoted to the computation of the velocity components, and the second loop reuses the velocity results of the previous time step to update the stress field.

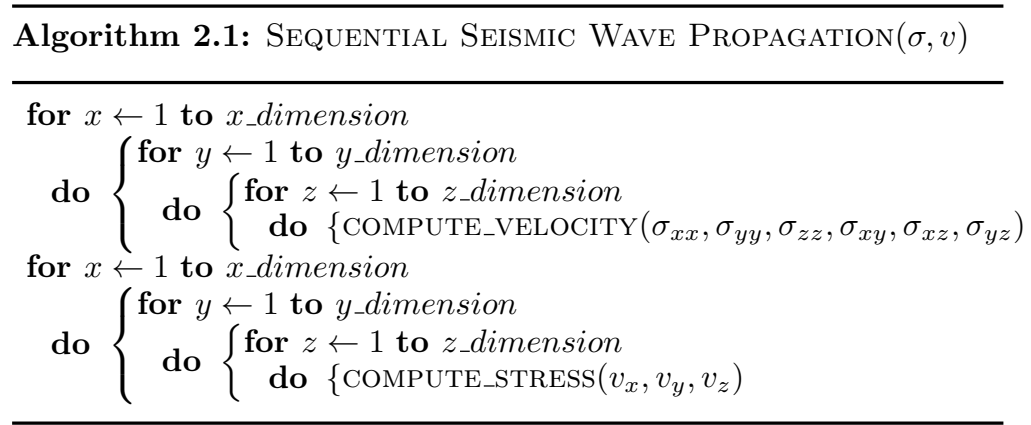

The best parallelization strategy for the elastodynamics equations strongly depends on the characteristics of the underlying hardware architecture. In the following section, we detail the parallelization and optimization strategies we employed for the adaptation of this sequential algorithm to highly parallel manycore processors.

\section{Elastodynamics Numerical Kernel on Manycores}

In this section we present our approaches to perform seismic wave propagation simulations on MPPA-256 and Xeon Phi. We first discuss in Section 3.1 some of the intrinsic characteristics and challenges that led us to employ different parallelization strategies and optimizations in each one of these processors. Then, in Sections 3.2 and 3.3, we describe in details our solutions for these hardware architectures.

\subsection{Platforms and Challenges}

MPPA-256. The MPPA-256 is a single-chip manycore processor developed by Kalray that integrates 256 user cores and 32 system cores in $28 \mathrm{~nm}$ CMOS technology running at $400 \mathrm{MHz}$. These cores are distributed across 16 compute clusters and $4 \mathrm{I} / \mathrm{O}$ subsystems that communicate through data and control NoCs. Each compute cluster has 16 cores called Processing Elements (PEs), which are dedicated to run user threads (one thread per PE) in non-interruptible and non-preemptible mode, and a low-latency shared memory of $2 \mathrm{MB}$. This local memory enables a high bandwidth and throughput between PEs within the same compute cluster. Each $\mathrm{PE}$ has private 2-way associative $32 \mathrm{kB}$ instruction and data caches. The system has no cache coherence protocol between PEs, even among those in the same compute cluster. The board used in our tests 


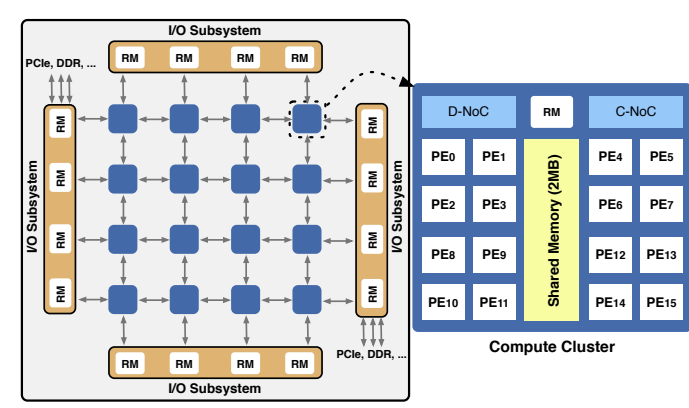

(a) MPPA-256.

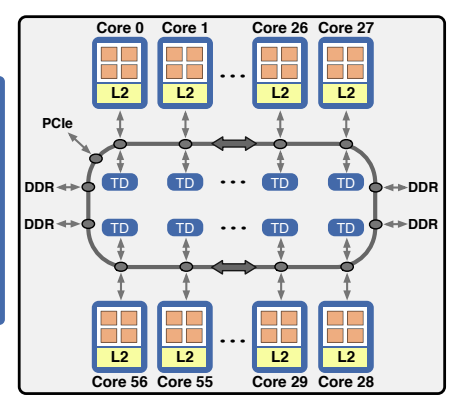

(b) Xeon Phi.

Figure 1: Manycore architectures.

has one of the I/O subsystems connected to an external LP-DDR3 of 2 GB. Figure 1(a) shows an architectural overview of the MPPA-256 processor. Some uses of this processor include embedded parallel signal processing and video decoding [21], and parallel scientific applications [7].

The development of a seismic wave propagation kernel for this processor can be a challenging task due to some of its intrinsic characteristics. First, the low-latency memory available in each compute cluster acts as a cache, whose goal is to store data retrieved from the DDR. However, data transfers between the DDR and compute clusters' low-latency memories must be explicitly managed by the programmer, in other words, there are no automatic fetching or prefetching mechanisms. Second, the amount of data needed to simulate real wave propagation scenarios does not fit into the local memories. In fact, about $500 \mathrm{kB}$ of memory is always in use by the operating system leaving about $1.5 \mathrm{MB}$ free to house the application's data and code. For this reason, the application has to take into account the data transfers and how these data should be sliced in order to fit into the limited local memory.

Xeon Phi. Xeon Phi was designed to operate in a way similar to regular x86 multicore processors. In this article we used Xeon Phi model 3120, which features 57 cores. Each one of its cores has a clock frequency of $1.10 \mathrm{GHz}$, $32 \mathrm{kB}$ for L1 instruction and data caches and $512 \mathrm{kB}$ for L2 cache. Coherence between the caches is guaranteed by hardware through a Global-distributed Tag Directory (TD). Additionally, every core can directly address the shared DDR memory (6 GB in our case) and is connected to the remaining cores by a high-performance bidirectional ring-shaped NoC as shown in Figure 1(b). Moreover, each core is 4-way multithreaded, i.e., it is able to execute instructions from four threads/processes (using time-multiplexed multithreading), helping to reduce the effect of vector pipeline latency and memory access latencies. Differently from other regular x86 multicore processors, each core has 512-bit vector instructions, which makes this processor able to perform 16 single precision operations, or 8 double precision operations, within a single instruction.

Porting a seismic finite difference numerical stencil to the Xeon Phi architecture requires a careful exploitation of two main aspects. The first one is related to the use of vector processing units. Since most of the performance power of 
Xeon Phi comes from these units, it is essential to fully benefit from them. This can be achieved by performing a clever decomposition of the 3D input problem to maximize the use of long vectors in the unit-stride direction. The second aspect is related to the L2 caches. When a core $C_{s r c}$ accesses its L2 cache and misses, an address request is sent to the tag directories throughout the ring. If the requested data block is found in the cache of another core $\left(C_{d s t}\right)$, it is forwarded back through the ring to the L2 cache of $C_{s r c}$. Thus, the overhead imposed by this protocol must be avoided whenever possible to improve application's performance. Overall, this can achieved by organizing data memory accesses to improve data locality.

In the next sections we describe how the architectural distinctiveness of these architectures guided the development of a seismic wave propagation simulation kernel.

\subsection{A Two-level Tiling Approach for the MPPA-256}

The seismic wave propagation kernel has a high demand for memory bandwidth. This makes the efficient use of the low-latency memories distributed among compute clusters indispensable. In contrast to standard $\mathrm{x} 86$ processors in which it is not uncommon to find last-level cache sizes of tens of megabytes, MPPA-256 has only $32 \mathrm{MB}$ of low-latency memory divided into $2 \mathrm{MB}$ chunks spread throughout the 16 compute clusters. These chunks of memory are directly exposed to the programmer that must explicitly control them. Indeed, the efficiency of our algorithm relies on the ability to fully exploit these lowlatency memories. To that end, we implemented a data fractioning strategy that decomposes the problem into tiles small enough to fit into the memory available on the compute clusters. Figure 2 illustrates the general idea of our two-level tiling scheme.

The three-dimensional structures corresponding to the velocity and stress fields are allocated on the DDR connected to the I/O subsystem to maximize the overall problem size that can be simulated. Next, we divide the global computational domain into several subdomains corresponding to the number of compute clusters involved in the computation (Figure 2-(1). This decomposition provides a first level of data-parallelism. To respect the width of the stencil (fourth-order), we maintain an overlap of two grid points in each direction. These regions, called ghost zones, are updated at each stage of the computation with point-to-point communications between neighboring clusters.

Unfortunately, only this first level of decomposition is not enough because the three-dimensional tiles do not fit into the $2 \mathrm{MB}$ of low-latency memories available to the compute clusters. A second level of decomposition is therefore required. We performed this decomposition along the vertical direction as we tile each three-dimensional subdomain into 2D slices (Figure 2-2). This leads to a significant reduction of the memory consumption for each cluster but requires maintaining a good balance between the computation and communication. Our solution relies on a sliding window algorithm that traverses the 3D domains using 2D planes and overlaps data transfers with computations. This mechanism can be seen as an explicit prefetching mechanism (Figure 2-3) as 


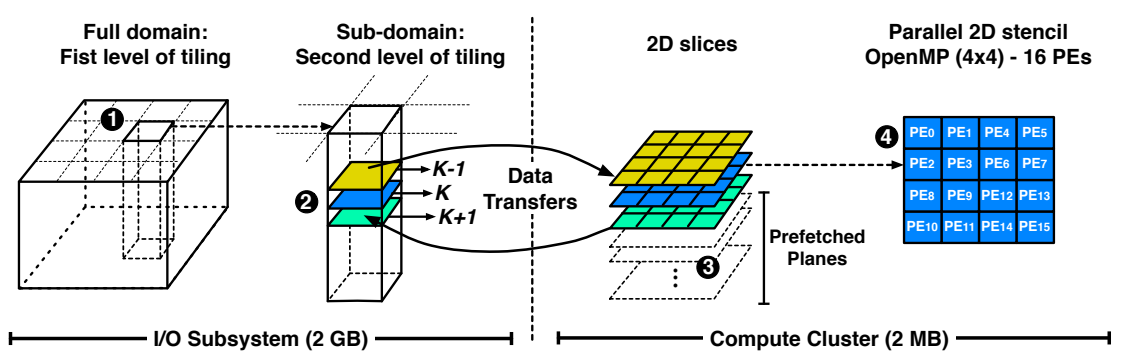

Figure 2: Two-level tiling scheme to exploit the memory hierarchy of MPPA-256.

2D planes required for the computation at one step are brought to the clusters during the computation performed at previous steps. To alleviate communication costs, asynchronous background data transfers are employed. Finally, OpenMP directives are employed inside each compute cluster to compute 2D problems with up to 16 PEs in parallel (Figure 2-4). Additional details about the implementation of the seismic wave propagation simulation kernel for the MPPA-256 architecture are provided by Castro et al. [8].

\subsection{A Cache-aware Approach for the Xeon Phi}

Research on the efficiency of seismic wave propagation simulations using the FDM on multicore architectures has received a lot of attention lately [22, 23]. The performance of these simulations are typically memory-bound due to the imbalance between the relatively fast point-wise computations and the intensive memory accesses these computations require.

Several standard sequential implementations offer a poor cache reuse and therefore achieve only a fraction of the peak performance of the processor. Even if some optimizations alleviate this problem, the standard simulation algorithm typically scans an array spanning several times the size of the cache using each retrieved grid point only for a few operations. Therefore, the cost to bring the needed data from the main memory to the fast local cache memories account for an important share of the total simulation time, specially for a 3D problem.

In particular, this limitation is more noticeable in processors that feature high core counts such as Xeon Phi due to the contention on the bidirectional data ring. To improve the cache locality we employ a cache blocking technique. Cache blocking is an optimization technique that intends to reduce memory bandwidth bottlenecks. The main idea is to exploit the inherent data reuse available in the triple nested loop of the elastodynamics kernel by ensuring that data remains in cache across multiple uses.

Among classical blocking strategies, the approach described by Rivera and Tseng [24] proposes to tile two dimensions and to perform computations by accumulating the layers in the third one. This strategy is somehow similar to our tiling strategy proposed for the MPPA-256, since the idea is to control the data movement by prefetching the read and the write computing planes.

Although the sliding window algorithm and vectorial instructions are a good combination, their use on Xeon Phi is unfeasible in practice. The limited cache 
size on Xeon Phi prevents the storage of three velocity and six stress components in $512 \mathrm{kB}$ of L2 cache memory shared by up to four threads. For that reason, our implementation therefore relies on a 3D blocking strategy which leads to a important speedup when we take the naïve implementation as a baseline as shown in Section 4. This improvement is due to the reduction of the pressure on the memory. We employ OpenMP to distribute the load throughout the different computing cores and we use the OpenMP's loop collapse directive to improve loop execution scheduling across the 3D domain decomposition. Additionally, we consider low-level specific optimizations such as data alignment, vectorization and thread affinity. On Xeon Phi, unaligned data severely limits the overall performance. In our implementation, we align data with respect to the length of the stencil (fourth order in our case) and we shift pointers in order fully benefit from the Xeon Phi vectorization capabilities (16 single precision floats). A similar optimization strategy can be found in [25]. Finally, we employ thread affinity to ensure that threads are correctly bound to the cores to reduce memory access latency and to alleviate the memory contention.

\section{Experimental Results}

In this section we evaluate the performance and energy consumption of our solutions to perform seismic wave propagation simulations on manycores. First, we briefly describe our evaluation methodology. Then, we analyze the impact of the optimizations on MPPA-256 and Xeon Phi. Finally, we carry out an overall comparison of the performance and energy consumption between our solutions for manycores and other standard optimized solutions for other modern platforms.

\subsection{Methodology}

We compare our solutions for the MPPA-256 and Xeon Phi to reference implementations of the same elastodynamics kernel for multicores $[8,26]$ and GPUs [8, 27]. These implementations were extracted from Ondes3D, a seismic wave propagation simulator developed by the French Geological Survey (BRGM). In this study we used the following platforms:

- MPPA-256: This low-power manycore processor integrates 16 compute clusters in a single chip running at $400 \mathrm{MHz}$. Each compute cluster has 16 cores called PEs and a low-latency shared memory of $2 \mathrm{MB}$ shared between all the PEs. Additionally, each PE has non-coherent, $8 \mathrm{kB}, 2$-way set associative caches, one for data and another for instructions. Compute clusters can communicate with each other and can indirectly access an external LP-DDR3 of 2 GB through data and control NoCs. Compilation was done using Kalray Compiler 9898489 (based on GCC 4.9) with the flags -03, -fopenmp, and -ffast-math.

- Xeon Phi: The processor used in this article features 57 4-way multithreaded cores. Each one of its cores has a clock frequency of $1.10 \mathrm{GHz}$, $32 \mathrm{kB}$ for L1 instruction and data caches and $512 \mathrm{kB}$ for L2 cache. Every 
core can directly address the shared DDR memory (6 GB) and is connected to the remaining cores by a high-performance bidirectional ring-shaped NoC. Intel's ICC version 14.0 was used to compile the code with the following compilation flags: -03, -openmp, -mmic (enables the cross compiler nedeed for a native execution on the Xeon Phi), and $-f p$-model fast $=2$. Additionally during development, we used the flag-vec-report 2 to verify whether the seismic wave simulation kernel was successfully vectorized.

- Tesla K20: This graphics board features NVIDIA Kepler architecture with 2496 CUDA parallel-processing cores, with working frequencies up to $758 \mathrm{MHz}$ and $5 \mathrm{~GB}$ of GDDR5 GPU memory. Compilation was done using NVIDIA's nvcc version 5.5 with the flags -03 -arch sm_20, and --use_fast_math, using GCC 4.9 as the host compiler.

- Xeon E5: This platform features a Xeon E5-4640 Sandy Bridge-EP processor, which has 8 physical cores, with working frequencies up to $2.4 \mathrm{GHz}$ and 32 GB of DDR3 memory. The code was compiled with GCC 4.9 with the flags -03, -fopenmp, and -ffast-math.

- Altix UV 2000: It is a Non-Uniform Memory Access (NUMA) platform composed of 24 NUMA nodes. Each node has a Xeon E5-4640 Sandy Bridge-EP processor (with the same specifications of the Xeon E5 platform) and 32 GB of DDR3 memory shared in a cc-NUMA fashion (NUMAlink6). Overall, this platform has 192 physical cores. Compiler version and flags are identical to that of the Xeon E5 platform.

We use four metrics to compare the energy and computing performance: time-to-solution, energy-to-solution, speedup and Energy-delay Product (EDP). Time-to-solution is the time spent to reach a solution for a seismic wave propagation simulation. Analogously, energy-to-solution is the amount of energy spent to reach a solution for a seismic wave propagation simulation. Speedups are calculated by dividing the time-to-solution of the sequential version by timeto-solution of the parallel/distributed version with $n$ threads. The EDP, initially proposed by Horowitz et al. [28], fuses the time-to-solution and energy-tosolution into a single metric, allowing hardware platforms to be directly compared taking into account both metrics. Since the relative weights between performance and energy consumption are subject to pragmatic concerns, we show the values for both EDP and Energy-delay-squared Product $\left(\mathrm{ED}^{2} \mathrm{P}\right)$ [29]. All results represent averages of 30 runs to guarantee statistically relevant values.

The energy-to-solution was obtained through each platform's specific power measurement sensors. Both Xeon E5 and Altix UV 2000 are based on Intel's Sandy Bridge microarchitecture. This microarchitecture has Intel's Running Average Power Limit (RAPL) interface which allows us to measure the power consumption of CPU-level components through hardware counters. We used this approach to obtain the energy consumption of the whole CPU package including cores and cache memory.

On Xeon Phi, we used Intel's MIC System Management and Configuration (MICSMC) tool which allows us to monitor the processor's power consumption. 
Power measurements obtained from RAPL and MICSMC are very accurate as shown in [30, 31]. Similarly, MPPA-256 features a tool called K1-POWER to collect energy measurements of the whole chip, including all clusters, on-chip memory, I/O subsystems and NoCs. According to Kalray's reference manuals, the measurement precision on MPPA-256 is $\pm 0.25 \mathrm{~W}$. NVIDIA Kepler GPUs such as Tesla K20 also have a similar tool called NVIDIA Management Library (NVML). We used the NVML to gather the power usage for the GPU and its associated circuitry (e.g., internal memory). According to NVML documentation, readings are accurate to within $\pm 5 \%$ of the actual power draw.

MPPA-256, Xeon Phi and Tesla K20 architectures can all be used as accelerators, where the main application code is executed on the host and performancecritical sections of the seismic wave propagation kernel are offloaded to them. However, since we intend to compare the performance and energy consumption of our seismic wave propagation solutions for these processors, we execute the entire application on MPPA-256 and Xeon Phi in native mode. In this case, both the main application code and the seismic wave propagation kernel are crosscompiled for them. GPUs, however, cannot be used in native mode. For that reason we avoid host intervention whenever possible. During our tests the host was only responsible for loading the kernel and for performing the initial and final data exchanges between the host and GPU memories. To provide a fair comparison and discard influences caused by the host, all the results presented in the following sections reflect only the execution of the kernel.

\section{2. $M P P A-256$}

Figure 3(a) shows the impact of the number of prefetched planes on communication and computation times. As we increase the number of planes available at the compute cluster memory level, we improve the reuse of data in the vertical direction. This allows us to overlap a considerable portion of data transfers with computations. However, we observed only slight improvements past six planes. This is due to the saturation of the NoC as this strategy increases the data traffic each time we increase the number of prefetched planes. Contrary to the other architectures, the small amount of memory available at each compute cluster on MPPA-256 (2 MB) obliges us to perform an important number of data transfers from/to the DDR. Due to the limited on-chip memory, were able to prefetch only up to eight planes before exhausting the available local memory in each compute cluster. Overall, the reason why the communication is still fairly high compared to computation is because the ratio between the time taken by a compute cluster to compute a subdomain of $1.5 \mathrm{MB}$ and the time taken to transfer 1.5 MB from/to the DDR is low. This limits the performance gains that can be achieved by overlapping communications with computations.

Figure 3(b) presents a weak scalability analysis of the seismic wave propagation kernel on MPPA-256 when we vary the number of prefetched planes and the number of clusters. For this analysis the problem size assigned to each cluster remains constant as we increase the number of clusters. Therefore, linear scaling is achieved if the normalized execution time is kept constant at 1.00 while the workload is increased in direct proportion to the number of clusters. 


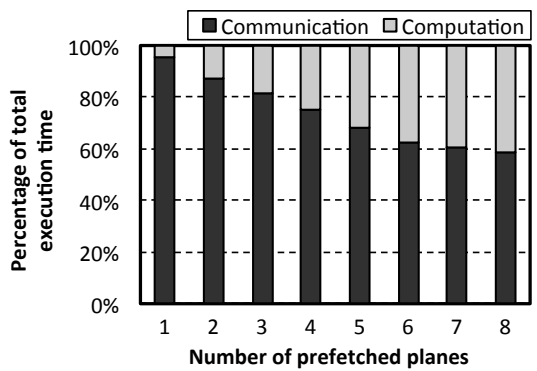

(a) Prefetching impact.

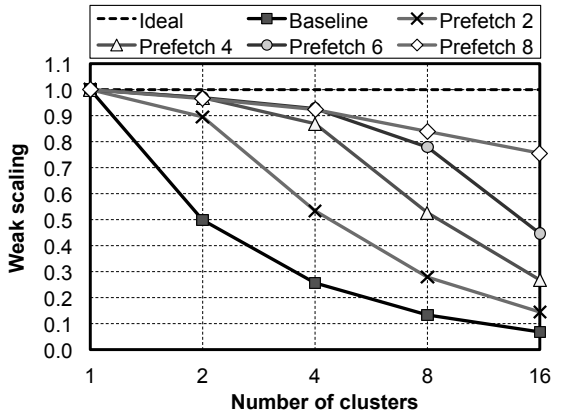

(b) Weak scalability.

Figure 3: Seismic wave propagation simulation kernel on MPPA-256.

Although the increase on the number of prefetched planes can indeed improve the scalability of our solution, there is still a considerable performance degradation as we employ an additional number of clusters. Even if our prefetching scheme is capable of masking communication costs on MPPA-256, the latency and bandwidth of the NoC can still hurt the execution performance and thus limit the scalability. A comparative scalability evaluation between MPPA-256 and Altix UV 2000 is presented in [8]. However, the results presented on that paper are slightly different from the ones we present here. This is due to the use of a newer toolchain in this work which has a slight performance improvement as well as better energy efficiency.

\subsection{Xeon Phi}

As discussed in Section 3.3, our solution to seismic wave propagation simulations on Xeon Phi employs not only the cache blocking technique, but also classic memory alignment with pointer shifting. Besides these optimizations, we experimented with different thread affinity and scheduling strategies.

Thread affinity can reduce variations on the execution time of an application and in some cases improve execution times by performing a better thread placement. We executed the seismic wave propagation kernel using the following OpenMP's thread placement policies: compact, scatter, balanced and default (no thread affinity). Setting affinity to compact will place OpenMP threads by filling cores one by one. Balanced affinity evenly distributes threads among the cores. It attempts to use all the available cores while keeping the thread neighboring logical IDs physically close to one another. The scatter affinity also evenly distributes threads among the cores but it does so in a round-robin fashion. Hence, threads with the adjacent IDs are not guaranteed to be physically adjacent.

Scheduling strategies dictate how the loop iterations are assigned to threads. We experimented with three scheduling strategies available in OpenMP: static, dynamic and guided. In static scheduling, the number of loop iterations is statically divided by the number of threads. This results in equal-sized chunks that are assigned to the threads (or as equal as possible in the case where the 


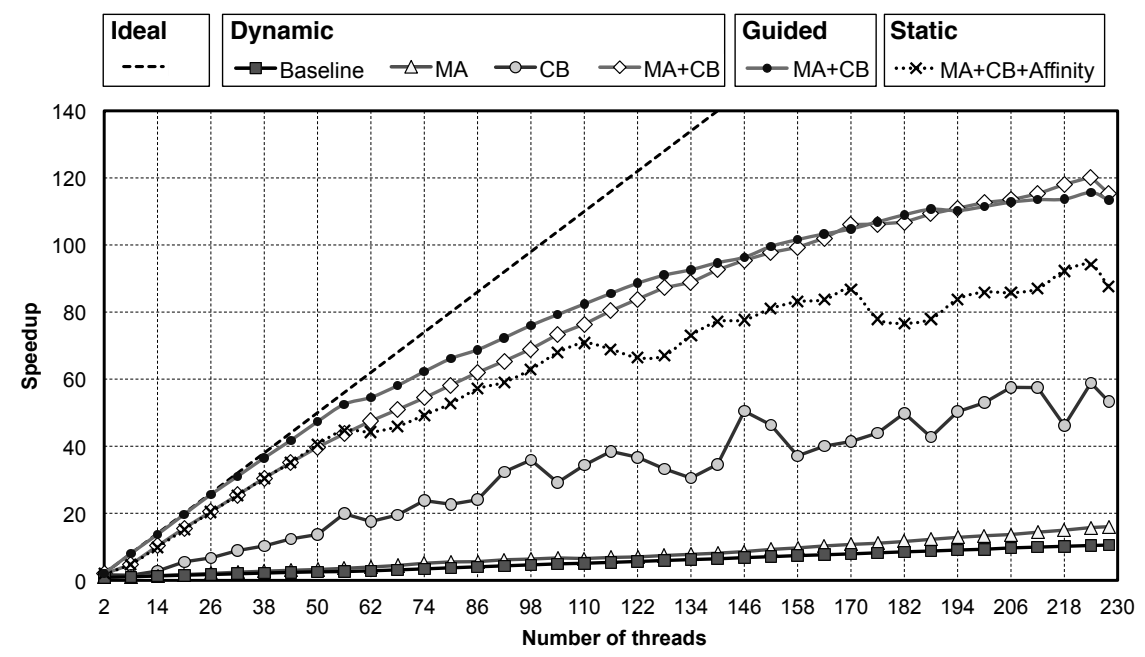

Figure 4: Impact of optimizations for dynamic and static OpenMP scheduling policies.

number of loop iterations is not evenly divisible by the number of threads). In dynamic scheduling, blocks of loop iterations are put into a shared work queue. These blocks are then dynamically distributed to the threads. When a thread finishes its block, it retrieves the next block of loop iterations from the top of the work queue. The size of the blocks is fixed and determined by the OpenMP's chunk parameter. The guided scheduling is similar to dynamic scheduling, but the size of the blocks starts off large and decreases to better handle load imbalance between iterations. In this case, the OpenMP's chunk parameter defines the approximate minimum size of the block.

Figure 4 shows the speedup we obtained when the kernel was executed with dynamic scheduling with no optimizations (Baseline); only memory alignment $(M A)$; only cache blocking $(C B)$; memory alignment and cache blocking $(M A+C B)$. Moreover, it presents the results obtained with static and guided scheduling policies when all optimizations are applied. The chunk size used for the experiments with dynamic and guided scheduling policies was 1 , since it presented the best results ${ }^{1}$. Thread affinity (Affinity) was only applied to static scheduling. As we will explain later on, thread affinity has almost no impact when OpenMP's dynamic or guided scheduling policies are used.

Experimental results showed us that memory alignment is essential to make a good use of Xeon Phi's 512-bit vector instructions. However, in order to do so, cache blocking is indispensable. In fact, memory alignment alone accounted for an average improvement of $24 \%$ in comparison to the baseline. The performance improvement of this optimization is severely limited by the poor utilization of the local caches. On the other hand, when compared to the baseline, a more

\footnotetext{
${ }^{1}$ Experiments with chunks greater than 1 exhibited higher load imbalances resulting in poorer performance.
} 
efficient utilization of the caches through cache blocking alone increased the performance by $79 \%$ on average. If used in isolation, cache blocking does not allow a full use of Xeon Phi's vectorization capabilities and creates additional cache faults due to unaligned memory accesses. By using these two techniques at once we can, at the same time, make a good utilization of Xeon Phi's vectorial capabilities and its fast local caches. Indeed, when these two techniques are used together we see an average improvement of $92 \%$ in performance, and an improvement of $91 \%$ for 224 threads.

Dynamic and guided scheduling policies had a better scaling behavior as well as better performance than static scheduling. On average, dynamic scheduling provided an increase of $14 \%$ over static scheduling. Static scheduling has the downside of causing imbalances during the execution of the simulation with an intermediate number of threads. For instance, for 58 threads, iterations would be divided equally between threads, but since a single core would execute two threads, it would actually end up executing twice as many iterations as the other cores. This behavior can clearly be seen as steps in the speedup graph. However, this assumption is not enough to effectively explain why the performance using static scheduling is not on par with (or better than) the performance of dynamic scheduling when the number of threads is a multiple of the number of cores.

The reason for that lies on the high usage levels of the NoC during the implicit synchronization between the calculation of the velocity and stress components of the stencil. For each timestep, the stress calculation loop only starts its execution after all the velocity components of the previous loop have finished (cf. Algorithm 2.1). This synchronization makes every thread to try to communicate at the same time at the start of each parallel loop. On the static scheduler, the concurrency for the NoC might introduce small delays for the beginning of the execution of each thread, essentially making the loop's critical path slightly longer. While by itself each one of these delays is not very significant, they accumulate at each timestep both at the stress and velocity calculation loops. Since the concurrency for the NoC also increases with the number of threads, the relative execution performance difference between the static and dynamic approaches also increases. This imbalance is compensated on the dynamic case, even if the dynamic scheduler has a higher overhead, because it is able to keep more threads active up to the end of each loop.

Guided scheduling also allows for a better work distribution and smaller imbalances than the static scheduler between threads at the end of each loop. However, the synchronization between velocity and stress loops and the small delay introduced during the initial phases of each loop still create slight imbalances at the end of both the stress and velocity loops of each timestep. Using the guided scheduler we were able to achieve near linear speedups up to the number of cores. After this point, the dynamic scheduler gradually approaches the guided performance to eventually beat it with the best execution time with 224 threads.

Finally, for static scheduling, thread affinity was able to improve the performance of $M A+C B$ in $11 \%$, on average. On the other hand, by its nature, dynamic and guided scheduling reduce the importance of thread affinity. Indeed, 
scatter and balanced thread placement policies presented very similar results in these cases. However, the compact thread placement policy decreased the performance with dynamic and guided scheduling by $22 \%$ on average, since it creates an important imbalance between cores, specially for low thread-counts. The loss in performance is reduced as the number of threads increases, from $50 \%$ for 40 threads to $0 \%$ for thread counts beyond 222 .

Since Xeon Phi does not offer Dynamic Voltage and Frequency Scaling (DVFS), the energy consumption on this platform is directly related to the execution time of the application. The best execution time was obtained with dynamic scheduling with 224 threads, providing the smallest energy-to-solution at $4.42 \mathrm{~kJ}$, and thus resulting in an improvement of $91 \%$ when compared to the baseline $(46.76 \mathrm{~kJ})$.

\subsection{Overall Energy and Performance Results}

In this section we compare the performance and energy consumption of our seismic wave propagation simulation kernel on MPPA-256 and Xeon Phi against other multicore and GPU reference implementations. On Xeon E5, we used the solution proposed by Dupros et al. [26], which employs OpenMP for the parallelization. On GPU we relied on the solution proposed by Michéa and Komatitsch [27], which is a state-of-the-art parallel solution for GPU-based seismic wave propagation simulation.

DVFS can make a significant difference in both performance and energy consumption. Although not available on the manycore processors we evaluated, it is available for the Xeon E5 and GPU platforms. Therefore, for these platforms we always show two measurements. The first ones, Xeon E5 $(2.4 \mathrm{GHz})$ and Tesla K20 $(758 \mathrm{MHz})$, represent the experimental results when their frequencies are optimized for performance, i.e., using their maximum working frequencies. The second ones, Xeon E5 $(1.6 \mathrm{GHz})$ and Tesla K20 $(705 \mathrm{MHz})$, are relative to the optimal energy consumption setting, which for this kernel was $1.6 \mathrm{GHz}$ and $705 \mathrm{MHz}$ on Xeon Phi and Tesla K20, respectively.

Figure 5 compares the time-to-solution and energy-to-solution across the processors using a problem size of $2 \mathrm{~GB}\left(180^{3}\right.$ grid points) and 500 time steps. For these experiments we used the optimal number of threads on each platform. With the exception of Xeon Phi (in which the best results were obtained with 224 threads), the thread count was equal to the number of physical cores of each processor. As shown in Figure 4, our solution for Xeon Phi keeps scaling considerably well past the 57 physical cores.

To the best of our knowledge, GPUs are among the most energy efficient platforms currently in use for seismic wave propagation simulation. Yet, our proposed solution on MPPA-256 achieves the best energy-to-solution among the analyzed processors, consuming $78 \%, 77 \%, 88 \%, 87 \%$ and $86 \%$ less energy than Tesla K20 (758 MHz), Tesla K20 (705 MHz), Xeon Phi, Xeon E5 $(2.4 \mathrm{GHz})$, and Xeon E5 $(1.6 \mathrm{GHz})$, respectively. On the other hand, when we consider the timeto-solution, MPPA-256 does not have the upper hand. In fact Tesla K20 had the best execution times among all the evaluated architectures. This result was, however, to be expected since the theoretical peak performance of Tesla K20 (3.5 TFLOPS in single precision) is at least $75 \%$ higher than that of the second 


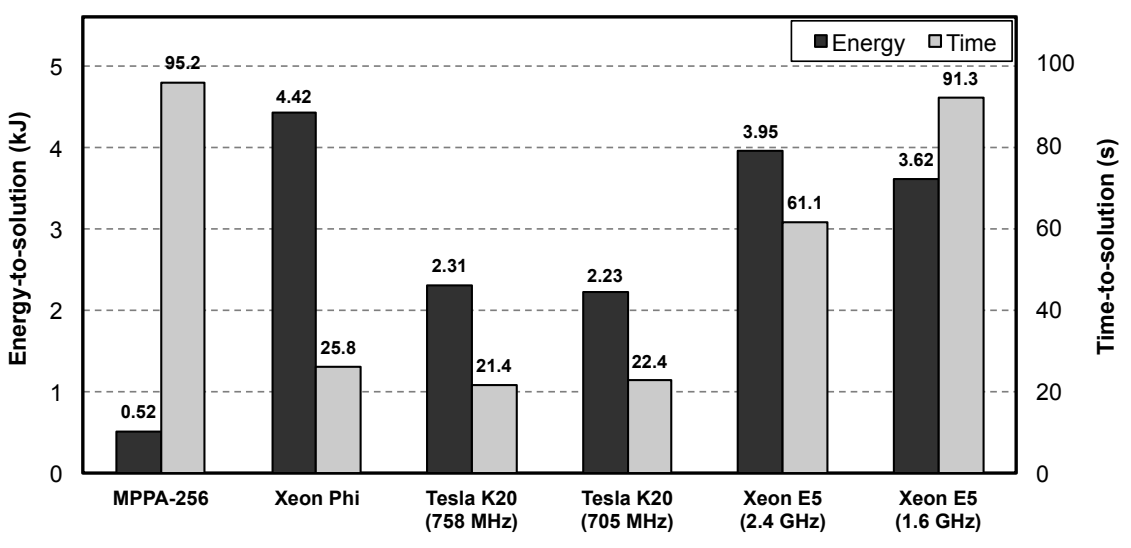

Figure 5: Chip-to-chip comparison.

placed architecture Xeon Phi (2.0 TFLOPS in single precision). Our solution for Xeon Phi improved the execution performance in $58 \%, 72 \%$ and $73 \%$ when compared to Xeon E5 $(2.4 \mathrm{GHz})$, Xeon E5 $(1.6 \mathrm{GHz})$ and MPPA-256, respectively. When compared to the Tesla K20 (758 MHz), our solution for Xeon Phi was only $20 \%$ slower, which not only is compatible to the nominal performance difference between these architectures, but also shows that our solution was able to provide hardware utilization levels that are at least as efficient as a state-of-the-art solution for GPUs.

A naïve direct comparison between the raw performance or the average power consumption between Altix UV 2000, a multi-processor architecture, to the previous single-processor architectures would make little sense. Therefore, we compare the Altix UV 2000 NUMA platform to the other processors in terms of energy efficiency and EDP. Table 1 shows the EDP and $\mathrm{ED}^{2} \mathrm{P}$ for all platforms (the lower the better). A scalability analysis of the seismic wave propagation kernel on Altix UV 2000 is discussed by Castro et al. [8].

When we consider EDP, MPPA-256 and Tesla K20 are clearly superior to the other evaluated platforms. If energy-to-solution alone is considered, MPPA256 has a clear advantage. MPPA-256 was conceived as a low-power highlyparallel processor. During our evaluation the MPPA-256 consumed on average only 6.45 W, while Tesla K20, Xeon Phi and consumed $184 \mathrm{~W}, 108 \mathrm{~W}$ and Altix UV $20001458 \mathrm{~W}$, respectively. However Tesla K20 shows a better balance between energy consumption and performance than the other manycore processors as evidenced by $\mathrm{ED}^{2} \mathrm{P}$. The high energy consumption of Xeon Phi places it right between MPPA-256/Tesla K20 and Xeon E5 when EDP is considered. However when we increase the time-to-solution weight $\left(\mathrm{ED}^{2} \mathrm{P}\right)$, Xeon Phi becomes more interesting than MPPA-256.

The Altix UV 2000 platform has the best trade-off between the time-tosolution and energy-to-solution, for both maximum performance and optimal energy consumption settings. If we consider $\mathrm{ED}^{2} \mathrm{P}$, this difference becomes even more noticeable. The significant differences highlighted in the combined time and energy evaluation can be explained by the poor arithmetic intensity 
Table 1: EDP (in thousands) for a simulation with $180^{3}$ grid points and 500 time steps.

\begin{tabular}{lrr}
\hline Platform & EDP & ED $^{\mathbf{2}} \mathbf{P}$ \\
\hline MPPA-256 & 49 & 4,695 \\
Xeon Phi & 114 & 2,934 \\
Tesla K20 $(758 \mathrm{MHz})$ & 49 & 1,056 \\
Tesla K20 $(705 \mathrm{MHz})$ & 50 & 1,124 \\
Xeon E5 $(2.4 \mathrm{GHz})$ & 242 & 14,755 \\
Xeon E5 $(1.6 \mathrm{GHz})$ & 330 & 30,143 \\
Altix UV 2000 $(2.4 \mathrm{GHz})$ & 13 & 40 \\
Altix UV 2000 $(1.6 \mathrm{GHz})$ & 17 & 74 \\
\hline
\end{tabular}

(FLOP/transferred byte ratio) which is characteristic of seismic wave kernels based on elastodynamics stencils. These memory-bound applications can experience important losses of performance if communications are not carefully overlapped with communications. On manycores such as MPPA-256 and Xeon Phi, there is an additional overhead created by the limited amount of fast local memory which forces the application to frequently employ the NoC during the execution. Indeed, Altix UV 2000 has approximately 20 times more fast local memory per thread than MPPA-256 and Xeon Phi. This reduces considerably the communication overhead associated to accesses to the main memory.

\section{Related Work}

Totoni et al. [6] compared the power and performance of Intel's Single-Chip Cloud Computer (SCC) to other types of CPUs and GPUs. The analysis was based on a set of parallel applications implemented with the Charm ++ programming model. Although they showed that there is no single solution that always achieves the best trade-off between power and performance, the results suggest that manycores are an opportunity for the future. Morari et al. [3] proposed an optimized implementation of radix sort for the Tilera TILEPro64 manycore processor. The results showed that the their solution for TILEPro64 provides much better energy efficiency than an general-purpose multicore processor (Intel Xeon W5590) and comparable energy efficiency with respect to a GPU NVIDIA Tesla C2070.

Francesquini et al. [7] evaluated three different classes of applications (CPUbound, memory-bound and mixed) using highly-parallel platforms such as MPPA256 and a 24-node, 192-core NUMA platform. They showed that manycore architectures can be very competitive, even if the application is irregular in nature. Their results showed that MPPA-256 may achieve better performance than a traditional general-purpose multicore processor (Intel Xeon E5-4640) on CPU-bound and mixed workloads whereas on a memory-bound workload Xeon E5 had better performance than MPPA-256. Among the evaluated platforms, MPPA-256 presented the best energy efficiency reducing the energy consumed on cpu-bound, mixed and memory-bound applications by at least $6.9 \mathrm{x}, 6.5 \mathrm{x}$ and $3.8 \mathrm{x}$, respectively. 
Using the low-power Adapteva Epiphany-IV manycore, Varghese et al. [5] described how a stencil-based solution to the anisotropic heat equation using a two-dimensional grid was developed. This manycore has a low power budged $(2 \mathrm{~W})$ and has 64 processing cores. Each core has a local scratchpad of $32 \mathrm{kB}$ and no cache-memory. Cores are connected by a NoC which allows direct memory access to $1 \mathrm{~GB}$ of RAM and also to other cores' local memories. The challenges we faced for the development of our seismic wave propagation simulation on the MPPA-256 architecture are similar to the ones faced by the authors of this work. Similar to MPPA-256, Epiphany-IV has a very limited amount of local memory available to each core and no automatic prefetching mechanism exists; every data movement has to be explicitly controlled by the application. The authors demonstrated that, even if the implementation of an efficient application can be a challenging task, their solution was able to achieve a FLOPS/Watt ratio 3 times better than an Intel 80-core Terascale Processor.

Adapting seismic wave propagation numerical kernels to emerging parallel architectures is an active research topic. Due the versatility of the FDM used both for oil and gas applications and standard earthquakes modeling, several strategies have been proposed. For instance, Intel and NVIDIA have proposed optimized implementations of such stencils [25, 32].

Dupros et al. [33] presented a review of the scalability issues for distributed and shared-memory platforms with a focus on mapping processes/threads on hierarchical clusters. Dursun et al. [34] introduced several additional strategies, including inter-node and intra-node optimizations. Recently, Christen et al. [35] described the use of the Patus framework to optimize the $A W P-O D C$ finite difference code. In particular, they detailed the impact of vectorization and cache prefetching and reported a performance improvement up to $15 \%$ when running the complete application with 512 MPI processes.

Several research efforts have been done on the adaptation of seismic wave kernels on GPUs to overcome the poor arithmetic intensity (FLOPS/transferred byte ratio) offered by elastodynamics stencils [27, 36]. The results obtained demonstrate that GPUs could be a relevant alternative to standard generalpurpose processors. Krueger et al. [37] compared the performance and energy efficiency of an Intel Nehalem platform, an NVIDIA Tesla GPU and a simulated general-purpose manycore chip design optimized for high-order wave equations called "Green Wave". They showed that Green Wave can be up to 8x and 3.5x more energy efficient per node when compared with the Nehalem and GPU platforms, respectively. Differently from this work, our experiments and measurements were carried out on real manycore processors. Even if the low-power MPPA-256 is not optimized for high-order wave equations as Green Wave, we were still able to achieve energy efficiency improvements of at least $86 \%$ and $77 \%$ when compared to the Xeon E5 and Tesla K20, respectively.

\section{Conclusion and Perspectives}

The use of highly-parallel manycore processors to meet the high demand for data processing capabilities needed by parallel scientific simulations has recently 
become one of central points of interest in the HPC community. In this paper, we presented our approach to the seismic wave propagation simulation using the MPPA-256 and Xeon Phi manycore processors. Although both processors share some architectural characteristics, they present different challenges that must be overcome in order to obtain good performance results.

The low-power MPPA-256 manycore processor has a limited amount of distributed low-latency memories that must be explicitly managed by the programmer. Xeon Phi, on the other hand, is a performance-centric manycore processor that requires careful source code changes to help the compiler to effectively vectorize time-consuming loops and to improve cache locality. In this article we proposed a new multi-level tiling strategy and a software prefetching mechanism that allowed us to perform real-world seismic wave propagation simulations and at the same time to lighten the communication overhead imposed by the NoC on the MPPA-256. On the other hand, on the Xeon Phi architecture we employed a cache blocking technique along with memory alignment and thread affinity strategies which allowed us to considerably improve the performance and scalability of our solution.

Our results showed that our approach to the MPPA-256 is the most energy efficient whereas our solution for the Xeon Phi achieves a performance comparable to the state-of-the-art solution for GPUs. MPPA-256 presented energy consumption improvements of $77 \%, 86 \%$ and $88 \%$ over other solutions for GPU, general-purpose multicore and the Xeon Phi manycore architectures, respectively. In terms of performance, on the other hand, our solution for Xeon Phi achieved performance improvements of $73 \%, 58 \%$ with respect to the solutions for MPPA256 and general-purpose multicore processor, respectively.

Despite encouraging results, our solutions for MPPA-256 and Xeon Phi manycore processors could still be improved. On MPPA-256, the communication still consumes a large amount of the total execution time $(58 \%)$. This is due to the high traffic on the NoC to access a single DDR memory. Kalray recently announced a multi-MPPA solution that features four MPPA-256 processors on the same board with less than $50 \mathrm{~W}$ of power consumption. In this new solution, each MPPA-256 processor can access two DDR3 channels in parallel and MPPA256 processors are interconnected through NoC eXpress interfaces (NoCX), providing an aggregate bandwidth of $40 \mathrm{~GB} / \mathrm{s}$. The benefits of this new solution for seismic wave propagation simulations are two-fold: (i) this would allow us to deal with input problem sizes of $32 \mathrm{~GB}$ or more; and (ii) distributing data among different MPPA-256 processors along with the parallel access to two DDR3 memories in each processor would alleviate the current communication bottleneck. Thus, we plan to work on new versions of our multi-level tiling strategy and prefetching scheme to exploit the full potential of multi-MPPA solutions as soon as they become available.

On Xeon Phi, the performance could still be improved by using an auto-tuning mechanism to automatically select the best size of the tiles and specific optimizations from the compiler based on the input problem and the number of threads used. Thus, we intend to study if frameworks such as Pochoir [38] or Patus [35] could provide us the essentials to implement the auto-tuning approach. 
Finally, we intend to study how our optimizations proposed in this paper could be made automatic. One possibility would be to extend PSkel [39], a stateof-the-art framework that provides a single high-level abstraction for stencil programming, to support MPPA-256 and Xeon Phi manycores. This would allow us to implement our proposed optimizations inside the framework, so applications implemented in PSkel will automatically benefit from software prefetching (MPPA-256) and cache blocking, memory alignment and thread affinity (Xeon Phi).

\section{Acknowledgments}

This work was supported by FAPESP grant 2014/17925-0, PNPD/CAPES, the European Community's Seventh Framework Programme (FP7/2007-2013) under the Mont-blanc 2 Project (www.montblanc-project.eu) grant 610402, and BRGM Carnot-institute. We would also like to thank Bull and the Center for Excellence in Parallel Programing (CEPP) for granting us access to their hardware infrastructure which was used in our experiments with Xeon Phi.

\section{References}

[1] N. Rajovic, N. Puzovic, L. Vilanova, C. Villavieja, A. Ramirez, The lowpower architecture approach towards exascale computing, in: Workshop on Scalable Algorithms for Large-scale Systems (ScalA), ACM, Seattle, USA, 2011, pp. 1-2.

[2] D. Göddeke, D. Komatitsch, M. Geveler, D. Ribbrock, N. Rajovic, N. Puzovic, A. Ramirez, Energy efficiency vs. performance of the numerical solution of PDEs: An application study on a low-power ARM-based cluster, Journal of Computational Physics 237 (2013) 132-150.

[3] A. Morari, A. Tumeo, O. Villa, S. Secchi, M. Valero, Efficient sorting on the Tilera manycore architecture, in: International Symposium on Computer Architecture and High Performance Computing (SBAC-PAD), IEEE Computer Society, New York, USA, 2012, pp. 171-178.

44 B. D. de Dinechin, P. G. de Massasa, G. Lagera et al., A distributed runtime environment for the Kalray MPPA-256 integrated manycore processor, in: International Conference on Computational Science (ICCS), Elsevier, Barcelona, Spain, 2013, pp. 1654-1663.

[5] A. Varghese, B. Edwards, G. Mitra, A. P. Rendell, Programming the Adapteva Epiphany 64-core network-on-chip coprocessor, in: International Parallel Distributed Processing Symposium Workshops (IPDPSW), IEEE Computer Society, Phoenix, USA, 2014, pp. 984-992.

[6] E. Totoni, B. Behzad, S. Ghike, J. Torrellas, Comparing the power and performance of Intel's SCC to state-of-the-art CPUs and GPUs, in: International Symposium on Performance Analysis of Systems and Software (ISPASS), IEEE Computer Society, New Brunswick, Canada, 2012, pp. 78-87. 
[7] E. Francesquini, M. Castro, , P. H. Penna, F. Dupros, H. C. de Freitas, P. O. A. Navaux, J.-F. Méhaut, On the energy efficiency and performance of irregular application executions on multicore, NUMA and manycore platforms, Journal of Parallel and Distributed Computing, In Press. doi:10.1016/j.jpdc.2014.11.002.

[8] M. Castro, F. Dupros, E. Francesquini, J.-F. Méhaut, P. O. A. Navaux, Energy efficient seismic wave propagation simulation on a low-power manycore processor, in: International Symposium on Computer Architecture and High Performance Computing (SBAC-PAD), IEEE Computer Society, Paris, France, 2014, pp. 57-64.

[9] E. Francesquini, A. Goldman, J.-F. Mehaut, A NUMA-Aware Runtime Environment for the Actor Model, in: International Conference on Parallel Processing (ICPP), IEEE, Lyon, France, 2013, pp. 250-259.

[10] L. L. Pilla, C. P. Ribeiro, D. Cordeiro, C. Mei, A. Bhatele, P. O. Navaux, F. Broquedis, J.-F. Mehaut, L. V. Kale, A hierarchical approach for load balancing on parallel multi-core systems, Proceedings of the 41st International Conference on Parallel Processing 0 (2012) 118-127.

[11] M.J. Rashti et al., Multi-core and network aware MPI topology functions, in: Proceedings of the 18th European MPI Users' Group conference on Recent advances in the message passing interface, EuroMPI'11, 2011, pp. 50-60.

[12] G. Mercier, J. Clet-Ortega, Towards an efficient process placement policy for mpi applications in multicore environments, in: Recent Advances in Parallel Virtual Machine and Message Passing Interface, Springer, 2009, pp. 104-115.

[13] J. McCalpin, Paleoseismology, 2nd Edition, Vol. 95, Academic press, 2009, Appendix 1.

[14] J. Lysmer, L. Drake, A finite element method for seismology, Methods in Computational Physics 11 (1972) 181-216.

[15] D. Komatitsch, J. P. Vilotte, The spectral-element method: An efficient tool to simulate the seismic response of $2 \mathrm{D}$ and 3D geological structures, Bulletin of the Seismological Society of America 88 (2) (1998) 368-392.

[16] M. Dumbser, M. Käser, An arbitrary high-order discontinuous Galerkin method for elastic waves on unstructured meshes II: The three-dimensional isotropic case, Geophys. J. Int. 167 (1) (2006) 319-336.

[17] J. Virieux, $P-S V$ wave propagation in heterogeneous media: Velocity-stress finite-difference method, Geophysics 51 (1986) 889-901.

[18] E. H. Saenger, N. Gold, S. A. Shapiro, Modeling the propagation of elastic waves using a modified finite-difference grid, Wave Motion 31 (1999) 77-92.

[19] W. Zhang, Z. Zhang, X. Chen, Three-dimensional elastic wave numerical modeling in the presence of surface topography by a collocated-grid finitedifference method on curvilinear grids, Geophysical Journal International 190 (1) (2012) 358-378.

[20] H. Aochi, T. Ulrich, A. Ducellier, F. Dupros, D. Michea, Finite difference simulations of seismic wave propagation for understanding earthquake 
physics and predicting ground motions: Advances and challenges, Journal of Physics: Conference Series 454 (2013) 1-9.

[21] P. Aubry, P.-E. Beaucamps et al., Extended cyclostatic dataflow program compilation and execution for an integrated manycore processor, in: Procedia Computer Science, Vol. 18, Elsevier, Barcelona, Spain, 2013, Ch. 2013 International Conference on Computational Science, pp. 1624-1633.

[22] K. Datta, S. Kamil, S. Williams, L. Oliker, J. Shalf, K. Yelick, Optimization and performance modeling of stencil computations on modern microprocessors, SIAM Review 51 (1) (2009) 129-159.

[23] M. Bianco, B. Cumming, A generic strategy for multi-stage stencils, in: International Conference on Parallel and Distributed Computing (EuroPar), ACM, Porto, Portugal, 2014, pp. 584-595.

[24] G. Rivera, C.-W. Tseng, Tiling optimizations for 3D scientific computations, in: Supercomputing Conferece (SC), ACM, Dallas, USA, 2000, pp. $32-38$.

[25] J. Reinders, J. Jeffers, High Performance Parallelism Pearls: Multicore and Many-core Programming Approaches, 1st Edition, Morgan Kaufmann, 2014.

[26] F. Dupros, C. Pousa, A. Carissimi, J.-F. Méhaut, Parallel simulations of seismic wave propagation on NUMA architectures, in: International Conference on Parallel Computing (ParCo), IOS Press, Lyon, France, 2010, pp. $67-74$.

[27] D. Michéa, D. Komatitsch, Accelerating a three-dimensional finitedifference wave propagation code using GPU graphics cards, Geophysical Journal International 182 (1) (2010) 389-402.

[28] M. Horowitz, T. Indermaur, R. Gonzalez, Low-power digital design, in: Low Power Electronics, 1994. Digest of Technical Papers., IEEE Symposium, 1994, pp. 8-11. doi:10.1109/LPE.1994.573184.

[29] A. J. Martin, Towards an energy complexity of computation, Information Processing Letters 77 (2-4) (2001) 181 - 187. doi:http://dx.doi.org/ 10.1016/S0020-0190(00)00214-3.

[30] M. Hähnel, B. Döbel, M. Völp, H. Härtig, Measuring energy consumption for short code paths using RAPL, ACM SIGMETRICS Performance Evaluation Review 40 (3) (2012) 13-17.

[31] G. Lawson, M. Sosonkina, Y. Shen, Energy evaluation for applications with different thread affinities on the Intel Xeon Phi, in: Workshop on Applications for Multi-Core Architectures (WAMCA), IEEE Computer Society, 2014, pp. 54-59.

[32] P. Micikevicius, 3D finite-difference computation on GPUs using CUDA, in: Workshop on General Purpose Processing on Graphics Processing Units, ACM, Washington, USA, 2009, pp. 79-84.

[33] F. Dupros, H.-T. Do, H. Aochi, On scalability issues of the elastodynamics equations on multicore platforms, in: International Conference on Computational Science (ICCS), Barcelona, Spain, 2013, pp. 1226-1234.

[34] H. Dursun, K. ichi Nomura, L. Peng et al., A multilevel parallelization 
framework for high-order stencil computations, in: International Conference on Parallel and Distributed Computing (Euro-Par), ACM, Delft, The Netherlands, 2009, pp. 642-653.

[35] M. Christen, O. Schenk, Y. Cui, PATUS for convenient high-performance stencils: evaluation in earthquake simulations, in: Supercomputing Conferece (SC), IEEE Computer Society, 2012, pp. 11:1-11:10.

[36] R. Abdelkhalek, H. Calandra, O. Coulaud, G. Latu, J. Roman, Fast seismic modeling and reverse time migration on a graphics processing unit cluster, Concurrency and Computation: Practice and Experience 24 (7) (2012) 739-750.

[37] J. Krueger, D. Donofrio, J. Shalf, M. Mohiyuddin, S. Williams, L. Oliker, F.-J. Pfreund, Hardware/software co-design for energy-efficient seismic modeling, in: Supercomputing Conferece (SC), IEEE Computer Society, 2011, pp. 73:1-73:12.

[38] Y. Tang, R. A. Chowdhury, B. C. Kuszmaul, C.-K. Luk, C. E. Leiserson, The pochoir stencil compiler, in: ACM Symposium on Parallelism in Algorithms and Architectures (SPAA), ACM, San Jose, USA, 2011, pp. 117-128.

[39] A. D. Pereira, L. Ramos, L. F. W. Góes, PSkel: A stencil programming framework for CPU-GPU systems, Concurrency and Computation: Practice and Experience.doi:10.1002/cpe.3479. 